\title{
Controlled release formulation of an anti-depression drug based on a L-phenylalanate- zinc layered hydroxide intercalation compound
}

\begin{abstract}
The intercalation of L-phenylalanate (LP) into the interlayer gallery of zinc layered hydroxide (ZLH) has been successfully executed using a simple direct reaction method. The synthesised intercalation compound, zinc layered hydroxide-L-phenylalanate (ZLH-LP), was characterised using PXRD, FTIR, CHNS, ICP-OES, TGA/DTG, FESEM and TEM. The PXRD patterns of the intercalation compound demonstrate an intense and symmetrical peak, indicating a well-ordered crystalline layered structure. The appearance of an intercalation peak at a low angle of $2 \theta$ with a basal spacing of $16.3 \AA$, signifies the successful intercalation of the L-phenylalanate anion into the interlayer gallery of the host. The intercalation is also validated by FTIR spectroscopy and CHNS elemental analysis. Thermogravimetric analysis confirms that the ZLH-LP intercalation compound has higher thermal stability than the pristine L-phenylalanine. The observed percentage of L-phenylalanate accumulated release varies in each release media, with $84.5 \%, 79.8 \%, 63.8 \%$ and $61.8 \%$ release in phosphate buffer saline (PBS) solution at $\mathrm{pH} 4.8$, deionised water, $\mathrm{PBS}$ solution at $\mathrm{pH} 7.4$ and $\mathrm{NaCl}$ solution, respectively. The release behaviour of LP from its intercalation compounds in deionised water and PBS solution at $\mathrm{pH} 4.8$ follows pseudo second order, whereas in $\mathrm{NaCl}$ solution and PBS solution at $\mathrm{pH} 7.4$, it follows the parabolic diffusion model. This study shows that the synthesised ZLH-LP intercalation compound can be used for the formation of a new generation of materials for targeted drug release with controlled release properties.
\end{abstract}

Keyword: Zinc layered hydroxides; Intercalation compounds; L-phenylalanine; Controlled release formulation 